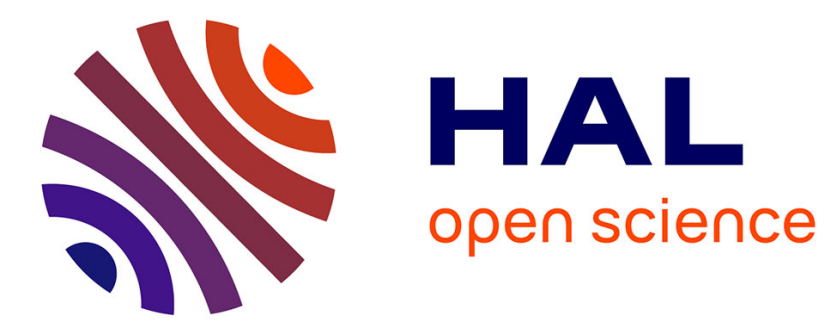

\title{
Il y a 400 ans, un roi renommé mais législateur forestier méconnu: Henri IV. \\ Jean-Luc Peyron
}

\section{To cite this version:}

Jean-Luc Peyron. Il y a 400 ans, un roi renommé mais législateur forestier méconnu: Henri IV..

Revue forestière française, 1997, 49 (5), pp.493-495. 10.4267/2042/5648 . hal-03444026

\section{HAL Id: hal-03444026 \\ https://hal.science/hal-03444026}

Submitted on 23 Nov 2021

HAL is a multi-disciplinary open access archive for the deposit and dissemination of scientific research documents, whether they are published or not. The documents may come from teaching and research institutions in France or abroad, or from public or private research centers.
L'archive ouverte pluridisciplinaire HAL, est destinée au dépôt et à la diffusion de documents scientifiques de niveau recherche, publiés ou non, émanant des établissements d'enseignement et de recherche français ou étrangers, des laboratoires publics ou privés. 


\section{II y a 400 ans, un roi renommé mais législateur forestier méconnu : Henri IV}

Henri IV, roi de France et de Navarre, occupe sans doute, dans le cœur des Français, une place tout à fait particulière, sinon exceptionnelle. Après la période troublée des guerres de religion, en dépit ou à la faveur d'un incontestable opportunisme, il symbolise celui qui a su tout à la fois reconstituer la France, en effectuer une remise en ordre, en assainir puis développer l'économie (Duby, 1971).

En effet, monté sur le trône en 1589, à l'âge de 36 ans, il lui faudra cinq ans pour accéder à sa capitale, Paris, près de dix ans pour faire entendre raison aux résistances intérieures (édit de Nantes, 13 avril 1598) et le même délai pour chasser les armées étrangères (traité de Vervins, 2 mai 1598).

Déjà amorcée pendant les années de lutte, la remise en ordre qu'il opère consiste à renforcer son pouvoir, à établir progressivement une monarchie centralisée, à pratiquer un jeu politique subtil fondé sur la recherche d'un consensus, nous dirions aujourd'hui la concertation. D'une certaine façon, les mesures forestières qu'il a été amené à prendre participent de cette remise en ordre.

Dans le domaine économique enfin, Henri IV soutient Sully dans son rétablissement des finances du royaume, suscite le lancement d'activités industrielles nouvelles, favorise le développement de la sériciculture lancée par Olivier de Serres, souscrit à l'assèchement des marais prôné par Laffemas.

Le succès de cette politique est clair aux yeux de la plupart des historiens qui n'hésitent pas à affirmer qu' "en une quinzaine d'années, les traces extérieures de la tourmente avaient disparu [pour conduire à une] impression de prospérité » (Duby et Wallon, 1975).

Aujourd'hui unanimement adulé, Henri IV a été largement controversé de son vivant. Son assassinat, en 1610, vient illustrer, s'il le fallait, cette contestation. C'est dire si son personnage et son action suscitent, de façon générale, les passions. Pourtant, au plan forestier, il est assez peu cité par les historiens, que ceux-ci soient généralistes ou spécialistes de ce domaine. Et, lorsqu'il l'est effectivement, c'est souvent pour se voir reprocher d'avoir puisé dans les forêts du royaume afin de renflouer les caisses de l'État. Badré (1983) cite ainsi un recours à la vente de bois consenti en 1601 pour subvenir aux frais de renouvellement d'une alliance avec les Suisses ; Bourgenot (1981) mentionne par ailleurs l'existence de coupes exceptionnelles en 1606 dans les hautes futaies de Laigue (900 arpents), de Compiègne (500 arpents) et de Retz (100 arpents) représentant environ 5 possibilités annuelles.

L'œuvre forestière d'Henri IV se limite-t-elle donc à ces coupes, à caractère exceptionnel, faisant d'ailleurs partie d'une stratégie globale cohérente élaborée en faveur de l'économie nationale ? Des éléments de réponse résident incontestablement dans l'ordonnance de Rouen de mai 1597, portant Règlement général des Eaux et Forêts, qui présente plusieurs intérêts. Quatre cents ans après, ceux-ci méritent d'être rappelés. 


\section{J.-L. PEYRON}

Ce Règlement général correspond tout d'abord à une remise en ordre des forêts qui en ont bien besoin à la fin de l'une des périodes les plus sombres de l'histoire de France (Badré, 1983). II commence par cette constatation : "les guerres civiles [...] ont tellement dépravé toutes choses, que tant par la negligence ou connivence de quelques-uns de nos Officiers, qu'effrenée licence ou impunité d'aucuns de nos subjets, lesdites forests sont presque ruinées entierement ». La situation déplorable dans laquelle se trouvent les forêts du royaume est donc la conséquence de multiples abus imputables à toutes catégories de personnes, y compris au sein de l'administration forestière elle-même. L'attitude du roi apparaît fort courageuse car elle est susceptible de lui attirer nombre d'opposants et d'ennuis. Badré (1983) explique ainsi que l'enregistrement de l'ordonnance ne se soit pas bien déroulé.

En ce qui concerne l'aménagement des forêts, le Règlement général de 1597 introduit, dès son article premier, une grande innovation dans l'organisation et l'assiette des coupes. Alors que des opérations d'arpentage étaient réalisées à l'occasion de chaque coupe, il est ordonné de "mesurer separement et borner la quantité d'arpens dont devra se faire vente par chacun an [...] \& par peintres estre faites cartes \& figures desdites forests, où seront denotées lesdites bornes ... ". Ainsi, des coupons sont-il assis une bonne fois pour toutes sur le terrain et sur les plans.

Dans ses articles II et III, le Règlement général enjoint aux responsables forestiers de visiter fréquemment les forêts placées sous leur responsabilité, tous les 6 mois pour les verdiers, gruyers, segrayers et maîtres sergents, le plus souvent possible pour les lieutenants et conseillers ès siège des tables de marbre. II demande ensuite de procéder, en prenant les forêts à tour de rôle, à des réformations, que nous appellerions aujourd'hui des aménagements. Le produit des amendes, confiscations et restitutions décidées à l'occasion des réformations vient couvrir le coût de ces dernières. Enfin, le Règlement général utilise, en précurseur, le terme de "mesnagement " en notant que les procès-verbaux des visites et réformations doivent être entreposés aux sièges des tables de marbre afin d'être utilisés " pour nostre interest bien \& mesnagement de nosdites Eaues \& Forests ".

Dans son article XXX consacré aux forêts des ecclésiastiques, commanderies et communautés, le Règlement rétablit le tiers en réserve qui avait été instauré en 1561, réduit au quart en 1576, supprimé en 1580, et qui sera réduit à nouveau au quart par l'ordonnance de 1669 (Baudrillart, 1823). Cette mesure sera sans doute la plus mal acceptée et Huffel (1926) lui attribue la cause des problèmes de mise en application qui frapperont ce texte.

Sur un plan maintenant plus général, cette ordonnance présente également un grand intérêt. Elle a en effet été élaborée dans un esprit totalement nouveau. À la suite de la convocation à Rouen d'une assemblée de notables à la fin de 1596 pour amorcer la remise en ordre du royaume, une commission chargée de préparer la réforme forestière est constituée, dont les travaux déboucheront en particulier sur l'ordonnance de mai 1597. Cette commission comprend des forestiers, parmi lesquels Pierre Clausse de Fleury, ancien Souverain Grand Maître et futur Surintendant des Eaux et Forêts. Le Règlement général possède ainsi un contenu directement inspiré par les forestiers.

Cette façon de procéder est caractéristique des méthodes d'Henri IV et Huffel (1926) se plaît à citer à ce sujet le discours prononcé par le roi devant l'assemblée des notables de Rouen : "Je ne vous ai point appelé, comme le faisaient mes prédécesseurs, pour vous faire approuver mes volontés. Je vous ai assemblés pour recevoir vos conseils, pour les croire, pour les suivre... ".

Après avoir noté le grand intérêt de cette ordonnance portant règlement général, il faut bien convenir que ce texte pose question quant à sa légalité. En effet, il n'a été enregistré qu'en janvier 1610, trois mois avant la mort d'Henri IV, et cette formalité n'a pas été accomplie à la Cour du Parlement mais au siège des Eaux et Forêts, à la table de marbre de Paris. D'ailleurs, Sainctyon (1610) ne le mentionne pas dans son recueil des édits et ordonnances des rois. Nous avons vu que le fait de désigner les responsables des abus passés, d'une part, et les réticences manifestées par les ecclé- 
siastiques pour la réhabilitation du tiers en réserve, d'autre part, sont deux explications possibles de cette étrangeté.

L'ambiguïté dans laquelle a été placé ce texte est sans doute à l'origine de la méconnaissance dont il fait l'objet. Elle ne signifie pas cependant que sa portée ait été nulle. Huffel (1926) constate que le Règlement général de 1597 "constitue un document de premier ordre pour l'histoire de nos méthodes forestières françaises». Badré (1983) qualifie cette ordonnance de capitale. Bourgenot (1981) commente quant à lui les conséquences de ce document en notant que, "malgré le retard pris par son enregistrement, l'excellent contenu technique du Règlement fut appliqué immédiatement par les officiers forestiers qui, dès 1597, commencèrent à établir, forêt par forêt, des règlements particuliers pour leur exploitation [et que] bien des forêts royales furent alors aménagées sous le règne de Henri IV».

II apparaît finalement remarquable que ce texte, qui semble bien avoir gardé un caractère officieux pendant plus d'une décennie, ait pu avoir autant d'effets, vraisemblablement en raison de la qualité du processus de son élaboration et de son inspiration par ceux-là mêmes qui devaient le mettre en œuvre.

Malheureusement, les abus contre lesquels Henri IV avait lutté reprirent après sa mort à la faveur de nouveaux troubles (au début des règnes de Louis XIII et Louis XIV notamment) et d'une moindre volonté politique. Le Règlement général de 1597 se trouve ainsi rehaussé a posteriori, symbolisant les efforts d'un roi courageux pour remettre en ordre les forêts du royaume et annonçant la future ordonnance de 1669 dont le retentissement sera beaucoup plus grand.

J.-L. PEYRON

\section{BIBLIOGRAPHIE}

BADRÉ (L.). - Histoire de la forêt française. - Paris : Éditions Arthaud, 1983. - 312 p.

BAUDRILLART (J.-J.). - Traité général des eaux et forêts, chasses et pêches. - Paris : M ${ }^{\text {me }}$ Huzard, Arthus Bertrand et Warée oncle, 1823.

BOURGENOT (L.) . - Histoire des eaux et forêts du XVle siècle à la fin de l'ancien régime (1515-1789). In : Histoire des forêts / Raymond Lefebvre et Louis Bourgenot. - Édition provisoire manuscrite. - Nancy : ENGREF, 1981. 273 p. (pp 70-273).

DUBY (G.). - Histoire de la France. Tome II : de 1348 à 1852. - Paris : Librairie Larousse, 1971. - 455 p.

DUBY (G.), WALLON (A.). - Histoire de la France rurale. Tome II : de 1340 à 1789. - Paris : Éditions du Seuil, 1975. $-624 p$.

HENRI IV. - Ordonnance portant règlement général des eaux et forêts, donnée au mois de mai 1597, registrée en la chambre des Eaux et Forêts le 27 janvier 1610. In : Loix forestières de France / Pecquet. - 1782. - pp. 451-476.

HUFFEL (G.). - Les Méthodes de l'aménagement forestier en France ; étude historique. - Nancy, Paris, Strasbourg : Imprimerie Berger-Levrault, 1926. - $231 \mathrm{p}$.

PECQUET. - Loix forestières de France, commentaire historique et raisonné sur l'ordonnance de 1669, les règlements antérieurs, et ceux qui l'ont suivie ? - Paris : Prault père, 1782. - Tome second, $534+214 \mathrm{p}$.

SAINCTYON. - Les Edicts et Ordonnances des Roys, Coustumes des provinces, Règlements, Arrests et Jugements notables des Eaues et Forests. - 1610 . 